\title{
A Study on the Secure Business Web Service Based on ebXML
}

\author{
Dongil Shin ${ }^{1}$, Dongkyoo Shin ${ }^{1 *}$, Baek-Ho Sung ${ }^{1}$, and Jun-Hong Song ${ }^{2}$ \\ ${ }^{1}$ Department of Computer Science and Engineering, Sejong University \\ 98, Kunja-Dong, Kwangjin-Ku, Seoul 143-747, Korea \\ \{dshin, shindk, guardia\} egce.sejong.ac.kr \\ ${ }^{2}$ Industrial Bank of Korea (IBK), 50, 2-ga Ulchi-ro, Chung-gu, Seoul, Korea \\ lovelysong@ibk.co.kr
}

\begin{abstract}
While there is tremendous e-business value in the ebXML (Electronic Business using eXtensible Markup Language), security remains an unsolved problem and one of the largest barriers to adoption. XML Security technologies that have been emerging recently have extensibility and flexibility that is suitable for security implementation such as encryption, digital signature, access control and authentication. In this paper, we propose secure business Web Service models based on ebXML that allow trading partners to securely exchange XML based business transactions by employing XML security technologies.
\end{abstract}

\section{Introduction}

XML Security technologies emerging recently have extensibility and flexibility suitable for security implementation such as encryption, digital signature, access control and authentication. They are recommended to be used in ebXML security implementations. XML security technologies such as XML digital signatures [2] and SAML (Security Assertion Markup Language) [4] can be exploited to solve this problem. XML Encryption [1] is also recommended to solve the loss of confidentiality problem. Also, XKMS (XML Key Management Specification) [3] is recommended by the ebXML security team for key management as a substitute for PKI. In this paper, we propose secure business Web Service models based on ebXML that allow trading partners to securely exchange XML based business transactions by employing XML security technologies.

\section{Secure Business Web Services Model Based on ebXML}

We propose two ebXML business scenarios ensuring the trust relationship within the real trading partners. The first scenario performs a user authentication and updates the $\mathrm{CPP}$ in the repository. The procedure for the first scenario is presented in the form of

* Correspondence author

N. Koch, P. Fraternali, and M. Wirsing (Eds.): ICWE 2004, LNCS 3140, pp. 575-576, 2004.

(C) Springer-Verlag Berlin Heidelberg 2004 
a sequence diagram in Figure 1, where each box in the diagram denotes a Web Service or an application program. The second scenario performs business transactions within the trading partners, where security requirements are satisfied by applying security modules to implement business processes, as shown in Figure 2. In these scenarios, each XML security is constructed as a Web Service, which follows the Web Services standards proposed by the W3C and OASIS [1,2,3,4].

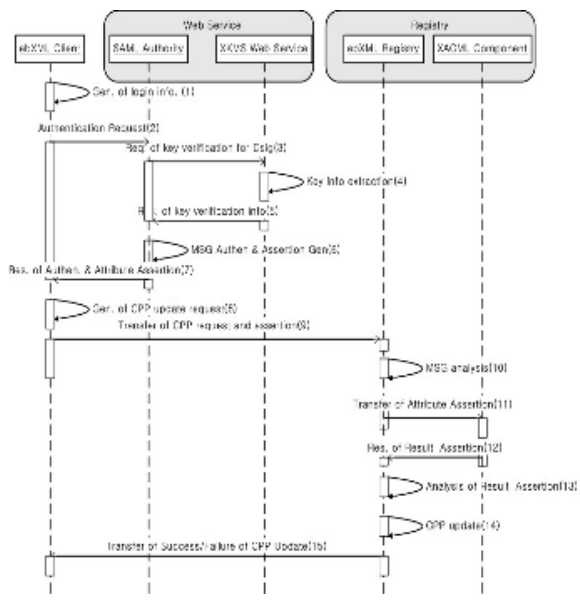

Fig. 1. Sequence Diagram - Senario 1

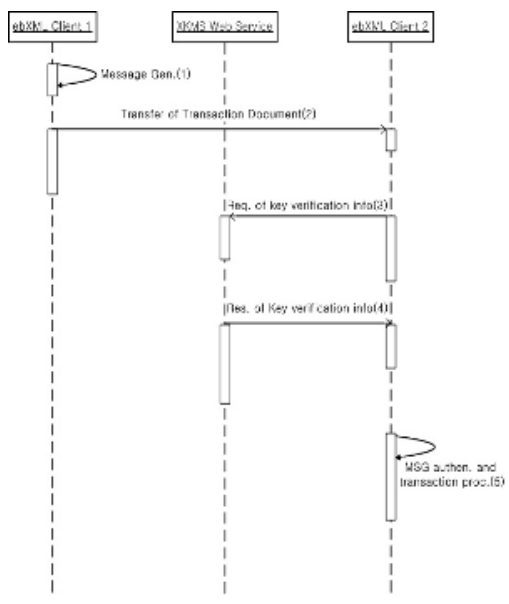

Fig. 2. Sequence Diagram - Senario 2

\section{Design and Implementation of the Test Software}

We constructed a test software, which focuses on security for registry/repository and messaging, and then targets system performance for the two business scenarios under a secure and reliable environment. XML Signature and XML Encryption are applied to the business transactions in the MSH (Message Service Handler) of ebXML client applications, registry, XKMS and SAML Web Services. We tested two scenarios by analyzing the messages in each step from Figure 1 and 2.

\section{References}

1. Imamura T., Dillaway B., Simon E.: XML Encryption Syntax and Processing, W3C Recommendation (2002), http://www.w3.org/TR/2002/REC-xmlenc-core-20021210/

2. Bartel M., Boyer J., Fox B., LaMacchia B. and Simon E.: XML Signature Syntax and Processing, W3C Recommendation (2002), http://www.w3.org/TR/xmldsig-core/

3. Ford W., Baker H., Fox B., Dillaway B., LaMacchia B., Epstein J. and Lapp J.: XML Key Management Specification (XKMS) Version 2.0, W3C Working Draft (2003), http://www.w3.org/TR/2003/WD-xkms2-20030418/

4. Maler E., Mishra P., Philpott R.: Assertions and Protocol for the OASIS Security Assertion Markup Language (SAML) V1.1, OASIS Committee Specification (2003)

http://www.oasis-open.org/committees/download.php/3406/oasis-sstc-saml-core-1.1.pdf 